\title{
Optimization of Prepectoral Breast Reconstruction
}

\author{
Roberto Cuomo ${ }^{a}$ Francesco Ruben Giardino ${ }^{a}$ Alessandro Neri ${ }^{b}$ \\ Giuseppe Nisi $^{\mathrm{a}}$ Cesare Brandi ${ }^{\mathrm{a}}$ Irene Zerini ${ }^{\mathrm{a}}$ Han Jingjian ${ }^{\mathrm{c}} \quad$ Luca Grimaldi $^{\mathrm{a}}$ \\ a Unit of Plastic and Reconstructive Surgery, S. Maria Alle Scotte Hospital, Department of Medicine, \\ Surgery, and Neuroscience, University of Siena, Siena, Italy; ${ }^{b}$ Unit of General and Oncological Surgery, \\ S. Maria Alle Scotte Hospital, Department of Medicine, Surgery, and Neuroscience, University of Siena, \\ Siena, Italy; ' Jining First People's Hospital, Jining Medical University, Jining City, PR China
}

\section{Keywords}

Breast reconstruction - Braxon - Acellular dermal matrix ·

Breast $\cdot$ Breast cancer

\begin{abstract}
Introduction: Acellular dermal matrix (ADM) were introduced in the early 2000s and more recently permitted new protocols for breast reconstruction allowing a short operative time with improved outcomes until the new musclesparing breast reconstruction proposed in 2014 using the Braxon ${ }^{\circledR}$ ADM. The aim of this research is to propose a technique to improve the aesthetic outcome using Braxon ADM with prepectoral implants. Materials and Methods: The enrolled patients were submitted to a nipple skin-sparing mastectomy leaving 1 additional centimeter of subcutis on the proximal part of the upper pole of the breast (see Surgical Technique). Aesthetic outcomes were compared to those obtained with traditional breast reconstruction with prosthesis and the Braxon ADM placed in the prepectoral space. Mean values of aesthetic outcomes were analyzed using the Student $t$ test, and the $\mathrm{K}$ test was used to analyze interobserver variability. Results: The overall aesthetic score was improved of $29.6 \%$ of the patients; in particular the most im-
\end{abstract}

proved score was that for contour (+98.01\%). Statistical significance was found for contour, upper pole definition, and total score average $(p<0.05)$. The $\mathrm{k}$ test showed interobserver variability with a good level of agreement on contour $(\mathrm{K}=$ 0.832). Conclusions: The proposed technique has allowed attainment of good results in terms of aesthetic outcomes. In a small sample we did not register any particular complications but we verified a better satisfaction on the evaluation of the aesthetic result (level of evidence: 3 ).

(c) 2020 S. Karger AG, Basel

\section{Introduction}

Breast cancer is one of the most frequents cancer in the world. It is the most common cancer in the UK, with 50,000 new cases diagnosed each year [1]. The American Cancer Society registered 63,410 in situ cases and 252,710 invasive cases in the USA in 2017 [2].

On the other hand, postmastectomy breast reconstruction is constantly evolving. Breast reconstruction was first described by Vincent Czerny in 1895 by transplantation of a lipoma taken from the patient's flank [3]; the most important historical moment happened when 


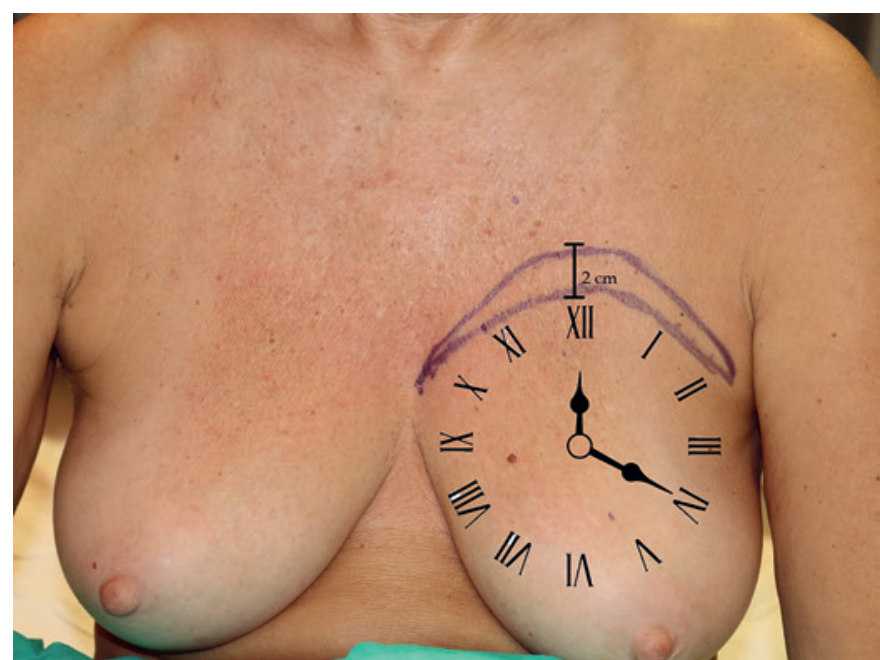

Fig. 1. Preoperative drawings.

Cronin implanted the first prosthesis in 1963 for aesthetic purposes, and, to date, many plastic surgeons choose an implant-based approach for breast reconstruction.

Acellular dermal matrix (ADM) was introduced in the early 2000s and more recently permitted new protocols for breast reconstruction allowing a short operative time with improved outcomes [4] until the new muscle-sparing breast reconstruction proposed by Berna et al. [5] in 2014 using the Braxon ${ }^{\circledR}$ ADM [5].

The main advantage of prepectoral implant for breast reconstruction consists of avoiding the complications and morbidity associated with pectoralis major detachment.

This technique is very recent and must be investigated again carefully. One of the first problems that may arise when using prepectoral prostheses is dimpling of the upper pole of the breast. This occurs due to thinning of the subcutaneous component during skin-sparing mastectomy.

The aim of this research is to propose a technique to improve aesthetical outcomes using Braxon ADM with prepectoral implants.

\section{Materials and Methods}

This prospective study was conducted in according with the Oncological Surgery Unit of the University of Siena from February 2018 to July 2018.

We included patients who were suitable for mastectomy and immediate breast reconstruction (IBR) with Braxon ADM and a prosthesis placed in the prepectoral space and fulfilled the following inclusion criteria:

- no radiotherapy needed after surgery for breast cancer,

- estimated breast volume $<550 \mathrm{~cm}^{3}$,

- good subcutaneous thickness ( $>1 \mathrm{~cm}$ measured by a pinch test at the upper and medial aspect of the breast),
Table 1. Patient characteristics

\begin{tabular}{rllll}
\hline Patient No. & $\begin{array}{l}\text { Age, } \\
\text { years }\end{array}$ & $\begin{array}{l}\text { Hospitalization, } \\
\text { days }\end{array}$ & Complications & Smoker \\
\hline 1 & 48 & 3 & None & Yes \\
2 & 52 & 3 & None & Yes \\
3 & 48 & 3 & None & No \\
4 & 53 & 3 & Seroma & No \\
5 & 55 & 3 & None & Yes \\
6 & 46 & 2 & None & No \\
7 & 49 & 4 & None & No \\
8 & 50 & 3 & None & No \\
9 & 52 & 3 & Seroma & Yes \\
10 & 50 & 4 & Seroma & No \\
11 & 55 & 3 & None & No \\
12 & 54 & 3 & None & No \\
13 & 54 & 3 & None & Yes \\
14 & 62 & 4 & None & No \\
\hline
\end{tabular}

- no BRCA mutations detected,

- abundance of fat and a small amount of parenchyma in the superior part of the upper pole of the breast, investigated with magnetic nuclear resonance, and

- cancer localized in the inferior pole of the breast

The enrolled patients were submitted to a nipple skin-sparing mastectomy leaving 1 additional centimeter of subcutis on the proximal part of the upper pole of the breast (see Surgical Technique) and aesthetic outcomes were compared to those obtained with traditional breast reconstruction with a prosthesis and Braxon ADM placed in the prepectoral space.

The analysis of aesthetic outcomes was done, blinded, by 2 plastic surgeons and 2 general surgeons not involved in the reconstruction process (see Data Collection).

\section{Patient Drawings}

Before surgery the contour of the breast was accurately defined through skin marks. In the enrolled patients, a mark shaped like a croissant with a downward concavity in the proximal portion of the upper pole of the breast was made where the skin of the breast becomes vertical and continues above with the clavicle. The 2 extreme tips of the croissant were placed laterally and medially following the contour of the breast at 2 o'clock and at 10 o'clock (Fig. 1).

\section{Surgical Technique}

All of the enrolled patients were submitted to nipple skin-sparing mastectomy with a lateral or periareolar approach. During the procedure, the surgeon detached the breast from the subcutaneous fat under the skin, leaving about $1 \mathrm{~cm}$ of tissue, but saved about 2 $\mathrm{cm}$ of subcutaneous fat in the area with the croissant-like mark in order to improve the fullness of the upper extremity of the superior pole.

The surgeon sent a fragment of subcutis of the upper extremity of the excised breast parenchyma to the Pathological Operative Unit for immediate histological examination to confirm the absence of breast parenchyma in order to ascertain the correct radicality of the mastectomy.

After the procedure and once the results of the histological examination had been obtained, the pectoralis muscle was left intact and the pocket was evaluated with a sizer in order to place a prosthesis of an adequate volume. We used a Braxon ADM sheet with 
Table 2. Mean panel of scores

\begin{tabular}{llllll}
\hline & Contour & $\begin{array}{l}\text { Placement of the } \\
\text { implant }\end{array}$ & Projection & $\begin{array}{l}\text { Upper pole } \\
\text { definition }\end{array}$ & \multicolumn{1}{c}{ Total } \\
New technique $(n=14$ patients) & 2 & 1.55 & 1.48 & 1.8 & 6.83 \\
Traditional technique $(n=16$ patients) & 1.01 & 1.54 & 1.5 & 1.22 & 5.27 \\
Change in scores, $\%$ & +98.01 & +0.65 & -1.35 & +47.5 & +29.6 \\
$p<0.05$ (Student's $t$ test) & Significant & Not significant & Not significant & Significant Significant \\
\hline
\end{tabular}

dimension of $30 \times 40 \mathrm{~cm}$ in order to place breast implants (anatomical type) of up to $550 \mathrm{~cm}^{3}$.

The ADM was rehydrated and prepared with sutures around the implant using $2 / 0$ vycril.

The wounds were closed using vycril $3 / 0$ for the subcutis and intradermal 3/0 monocryl plus cyanoacrylate glue [6].

\section{Data Collection}

We collected the age of the patients, as well as their smoking status, time of hospitalization, and complications (infections, seroma, loss of implants, and wound diastases) (Table 1).

\section{Assessment of Aesthetic Outcomes}

Aesthetic outcomes were evaluated by 2 oncological surgeons and 2 plastic surgeons not involved in the operative plan, in a blinded way (i.e., the doctor did not know if patient was in the group of enrolled patients or not). The 4 medical doctors analyzed 5 pictures of each patient in lateral (right and left), oblique (right and left), and frontal views. The pictures were submitted at 10 days, 3 months, and 6 months postoperatively.

We asked the doctors to evaluate the contour, the placement of the implant, projection, and upper pole definition according to a modified grading scale proposed by Garbay et al. [7] and modified by Ibrahim et al. [8] on a scale of $0-2$ ( 0 for the worst outcome and 2 for the best outcome), so that all the surgeons wrote a single value for each item and for each patient; all of these items values were grouped and averaged. The overall score was obtained by totaling the subscale averages for each patient.

The average values obtained from the surgeons about the aesthetic aspect were stratified with Student's $t$ test and the variability was analyzed with the Fleiss $\kappa$ test.

Statistical analysis was performed using SPSS version 21 for Windows (Chicago, IL, USA).

\section{Results}

Fourteen patients were enrolled and treated with this technique, leaving $1 \mathrm{~cm}$ more than the normal of subcutaneous fat in the extreme portion of the upper pole. No patient reported infection of the implant or the surgical site, and 3 patients reported a seroma around the implant, placed at follow-up with ultrasound checks. All 3 seromas resolved spontaneously in 3 weeks without a trace. Pathological examination of subcutis specimens showed the absence of breast parenchyma in 13 cases. In 1 case a fragment of breast parenchyma was identified on the lower side of the specimen. In no case were neoplastic cells found.
The overall aesthetic score was improved (i.e., 29.6\%); in particular the most improved score was that for the contour $(+98.01 \%)$. Statistical significance was found for contour, upper pole definition, and for total score average $(p<0.05$; Table 2$)$.

The $\kappa$ test showed interobserver variability with a good level of agreement for contour $(\kappa=0.832)$ (Fig. 2-4).

\section{Discussion}

Breast reconstruction has changed over time and is still changing $[9,10]$. A few years ago IBR was used to indicate the implant of tissue expander after mastectomy. Recently the definition of IBR underwent a change, indicating immediate reconstruction and immediate placement of a breast implant under the muscular plane with or without the use of ADM. In these cases the use of ADM provides the appropriate implant cover and it is useful in larger anatomical breast pockets and in order to obtain better control of the inframammary fold [11]. Although the majority of IBR are performed by placing the implant in the subpectoral space, detachment and elevation of the pectoralis major muscle can cause deformity, discomfort, and pain and these aspects are a cause for concern among patients and surgeons [12-14]. Some researchers agree that the pain and discomfort levels are high and many patients need opioids during the $24 \mathrm{~h}$ following breast reconstruction in the subpectoral space $[11,14]$. Placement of implants in the subcutaneous pocket avoids stretching of the muscle with a reduction of pain. Sbitany and Langstein [15] studied the impact of ADM on IBR and concluded that the acellular derma matrixes improve the aesthetic and physiological outcomes in breast reconstruction when used to cover the prosthesis in the submuscular pocket. The use of ADM in breast reconstruction was analyzed by many authors to understand the incidence of complications and the aesthetic outcome in IBR and in delay breast reconstruction. Hoppe et al. [16], for example, proposed a meta-analysis that showed that the use of ADM yielded superior aesthetic results, but they founded higher rates of postoperative complications such as infections, seromas, and 


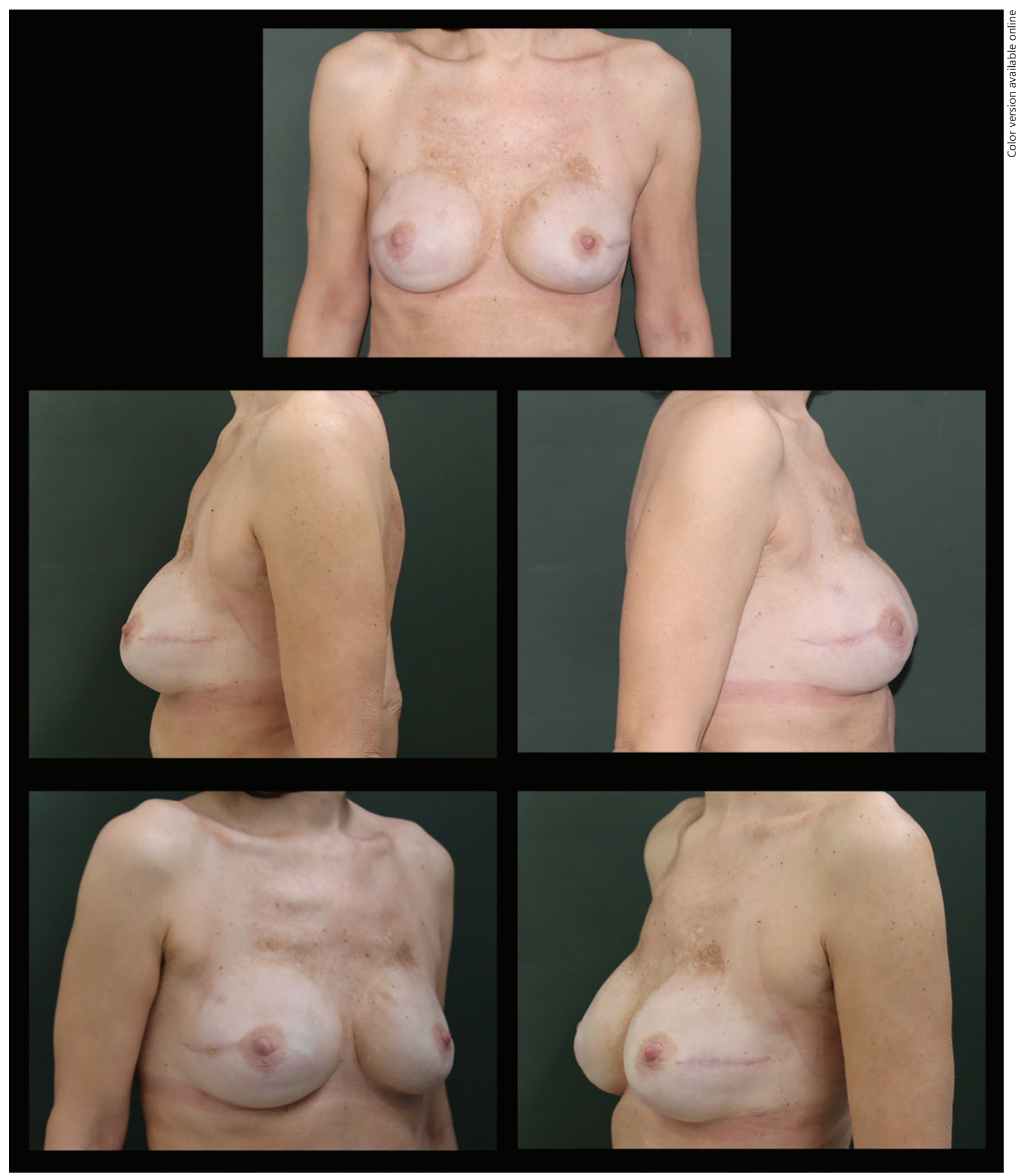

Fig. 2. Image of a patient operated with a traditional technique at 3 months of follow-up. In the upper pole of the breast the contour of the prosthesis is visible. 


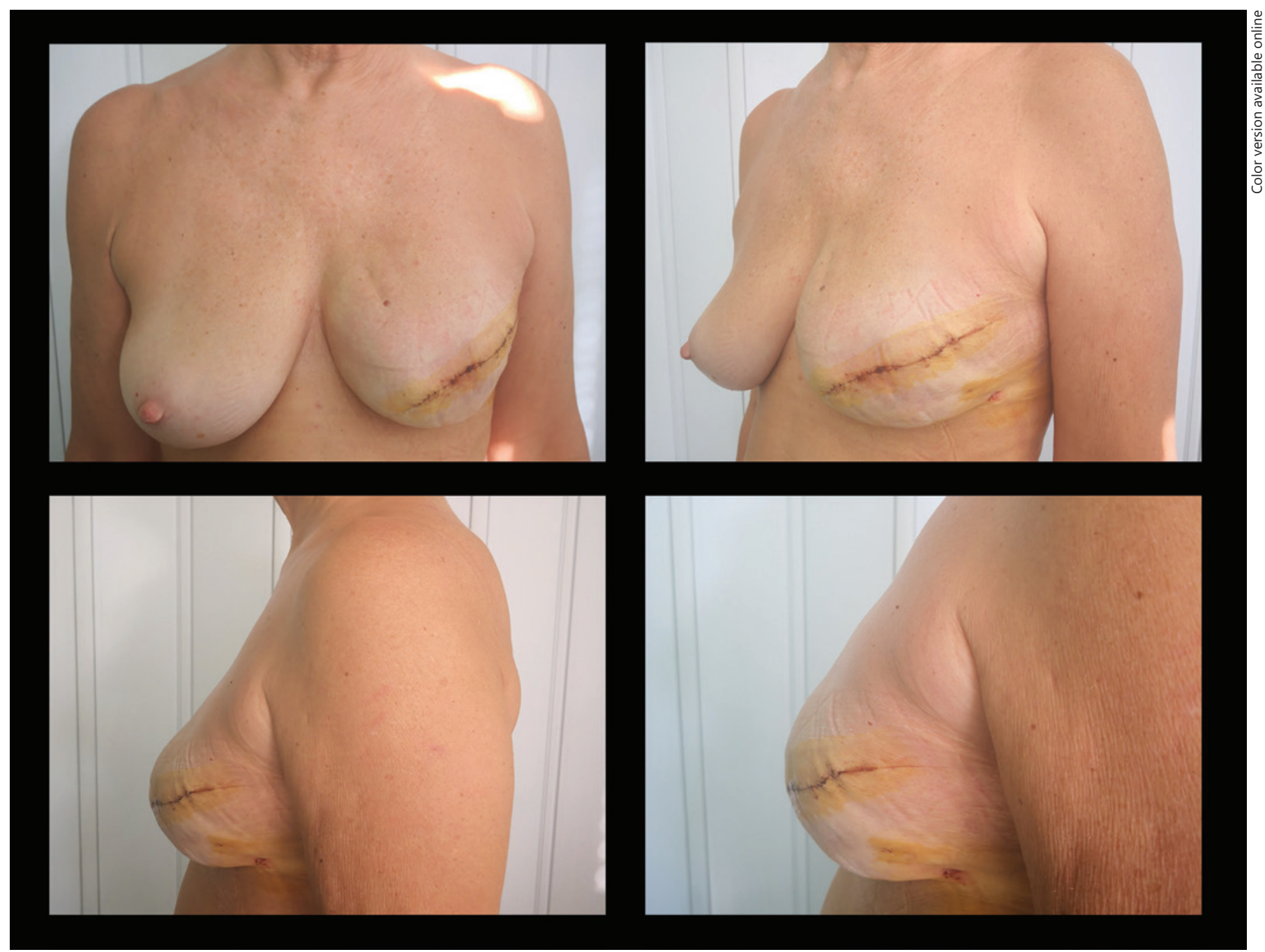

Fig. 3. New technique with good aesthetic outcomes with zoom in the lateral view.

implant loss. They concluded that expander/implant reconstruction results in increased rates of hematoma, seroma, infections, and loss of the implant because ADM is a foreign body which increases the inflammatory reaction and rates of infection compared to the use of an expander only. During the first weeks following the surgery, ADM does not have ingrowth of the vascular structure and in that period ADM is potentially susceptible to infections like with the expander $[16,17]$.

Surgeons now have the possibility to use ADM positioning breast implants in the prepectoral space by using Braxon ADM. This is a new technology and a topic of interest in the literature. The issue of detachment of the pectoralis major is solved by implanting a prosthesis in the prepectoral space. This technique is quite recent, but there is a general consensus regarding its use, with good reconstructive and aesthetic results. However, several authors agree that several measures are still needed to improve the final result $[9,18,19]$. One of the main issues with this new technique is the absence of pectoralis muscle on the implant, and this leads to the absence of a soft tissue buffer exacerbating the upper pole hollowing. This aspect was analyzed by Fracol et al. [18], who proposed a myotomy and capsulotomy with intramuscular fat grafting of about $100 \mathrm{ml}$ of fat per side in order to camouflage the implant edge. However, we are convinced that the great advantage of the use of $\mathrm{ADM}$ in the prepectoral region is keeping the pectoralis major muscle whole, so that this must not be cut or damaged with a fat graft.

It is also possible to obtain a good result by programming a good dissection, leaving a slightly larger quantity of adipose tissue (just $1 \mathrm{~cm}$ ) in the highest portion of the upper pole of the breast.
Cuomo/Giardino/Neri/Nisi/Brandi/ Zerini/Jingjian/Grimaldi 


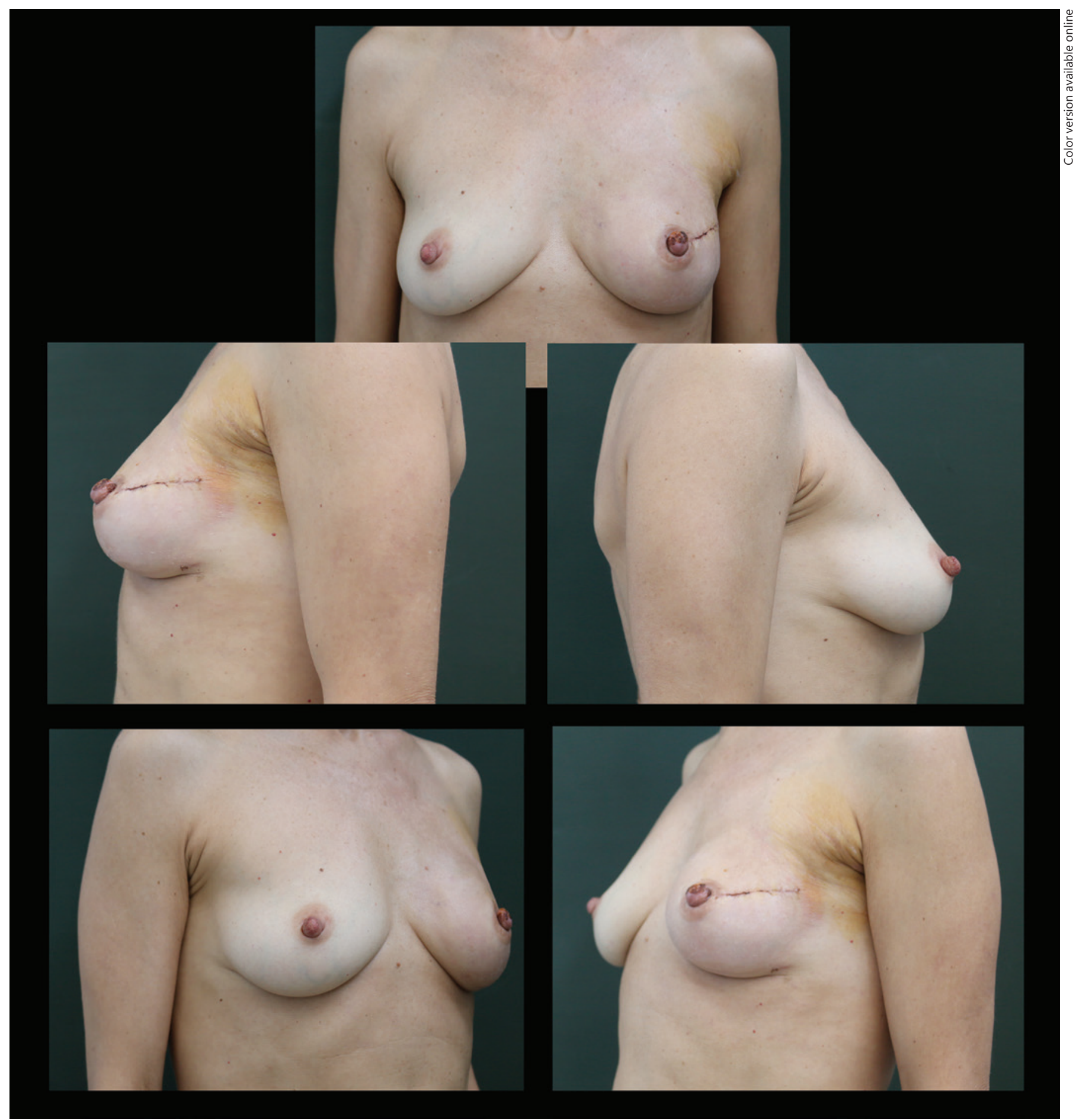

Fig. 4. New technique with good aesthetic outcomes in frontal, oblique, and lateral views at 3 months of follow-up.

\section{Conclusions}

Breast reconstruction with a prepectoral implant with a prosthesis and ADM allows attainment of very interesting, results although some problems with aesthetic outcomes have yet to be solved.

The proposed technique has yielded good results in terms of aesthetic outcomes, respecting the Best Practice
Guidelines (a specimen of subcutis was always analyzed). In a small sample, we did not register any particular complications but we verified a higher satisfaction on the evaluation of the aesthetic result. However, accurate preliminary planning is mandatory, but we believe that this technique could eventually be performed in a large percentage of patients undergoing mastectomy and reconstruction with a prepectoral system. 


\section{Statement of Ethics}

This study is in accordance with the Declaration of Helsinki. All of the patients signed an informed consent form and the ethical committee approved this research.

\section{Disclosure Statement}

The authors have nothing to declare.

\section{Funding Sources}

No funding was received.

\section{Author Contributions}

Dr. Cuomo proposed the technique and wrote this paper. Dr. Cuomo, Prof. Grimaldi, Prof. Neri, and Dr. Zerini performed the surgery. Dr. Cuomo, Prof. Nisi, Prof. Brandi, Dr. Jingian, and Dr. Giardino did the data collection, statistic processing.

\section{References}

1 Vidya R, Cawthorn SJ. Muscle-Sparing ADMAssisted Breast Reconstruction Technique Using Complete Breast Implant Coverage: A Dual-Institute UK-Based Experience. Breast Care (Basel). 2017 Sep;12(4):251-4.

2 American Cancer Society. Breast cancer facts \& figures 2017-2018 [Internet]. 2017. Available from: https://www.cancer.org/content/ dam/cancer-org/research/cancer-facts-andstatistics/breast-cancer-facts-and-figures/ breast-cancer-facts-and-figures-2017-2018. pdf.

3 Uroskie TW, Colen LB. History of breast reconstruction. Semin Plast Surg. 2004 May; 18(2):65-9.

4 Breuing KH, Warren SM. Immediate bilateral breast reconstruction with implants and inferolateral AlloDerm slings. Ann Plast Surg. 2005 Sep;55(3):232-9.

5 Berna G, Cawthorn SJ, Papaccio G, Balestrieri $\mathrm{N}$. Evaluation of a novel breast reconstruction technique using the Braxon ${ }^{\circledR}$ acellular dermal matrix: a new muscle-sparing breast reconstruction. ANZ J Surg. 2017 Jun;87(6):493-8.

6 Grimaldi L, Cuomo R, Brandi C, Botteri G, Nisi G, D'Aniello C. Octyl-2-cyanoacrylate adhesive for skin closure: eight years experience. In Vivo. 2015 Jan-Feb;29(1):145-8.
7 Garbay JR, Rietjens M, Petit JY. Esthetic results of breast reconstruction after amputation for cancer. 323 cases. J Gynecol Obstet Biol Reprod (Paris). 1992;21(4):405-12.

8 Ibrahim AM, Koolen PG, Ganor O, Markarian MK, Tobias AM, Lee BT, et al. Does acellular dermal matrix really improve aesthetic outcome in tissue expander/implant-based breast reconstruction? Aesthetic Plast Surg. 2015 Jun;39(3):359-68.

9 Stocco C, Figus A, Razzano S. Response to letter commenting on upgrading the BREAST$\mathrm{Q}$ questionnaire. J Plast Reconstr Aesthet Surg. 2018 Aug;71(8):1216-30

10 Stocco C, Figus A, Razzano S. Upgrading the BREAST-Q questionnaire with donor site evaluation after PAP flap breast reconstruction. J Plast Reconstr Aesthet Surg. 2018 Jun; 71(6):928-9.

11 Jafferbhoy S, Chandarana M, Houlihan M, Parmeshwar R, Narayanan S, Soumian S, et al. Early multicentre experience of pre-pectoral implant based immediate breast reconstruction using Braxon ${ }^{\circledR}$. Gland Surg. 2017 Dec; 6(6):682-8.

12 Hammond DC, Schmitt WP, O'Connor EA. Treatment of breast animation deformity in implant-based reconstruction with pocket change to the subcutaneous position. Plast Reconstr Surg. 2015 Jun;135(6):1540-4.
13 Spear SL, Schwartz J, Dayan JH, Clemens MW. Outcome assessment of breast distortion following submuscular breast augmentation. Aesthetic Plast Surg. 2009 Jan;33(1):448.

14 Cuomo R, Zerini I, Botteri G, Barberi L, Nisi G, D'Aniello C. Postsurgical pain related to breast implant: reduction with lipofilling procedure. In Vivo. 2014 Sep-Oct;28(5):993-6.

15 Sbitany H, Langstein HN. Acellular dermal matrix in primary breast reconstruction. Aesthet Surg J. 2011 Sep;31(7 Suppl):30S-7S.

16 Hoppe IC, Yueh JH, Wei CH, Ahuja NK, Patel PP, Datiashvili RO. Complications following expander/implant breast reconstruction utilizing acellular dermal matrix: a systematic review and meta-analysis. Eplasty. 2011;11:e40.

17 Nicoletti G, Saler M, Pellegatta T, Tresoldi MM, Bonfanti V, Malovini A, et al. Ex vivo regenerative effects of a spring water. Biomed Rep. 2017 Dec;7(6):508-14.

18 Fracol M, Qiu CS, Feld LN, Chiu WK, Kim JY. Myotomy-capsulotomy with intramuscular fat grafting: a novel technique for secondary treatment of prepectoral upper pole defects in breast reconstruction. Aesthet Surg J. 2019 Mar 14;39(4):454-9.

19 Nicoletti G, Scevola S, Ruggiero R, Coghi AM, Toussoun GS. Gigantic Paget disease of the breast. Breast. 2004 Oct;13(5):425-7. 